\title{
Development of a New Building Block \\ for the Synthesis of Silicon-Based Drugs and Odorants: \\ Alternative Synthesis of the Retinoid Agonist Disila-bexarotene
}

\begin{abstract}
Matthias W. Büttner, Jennifer B. Nätscher, Christian Burschka, and Reinhold Tacke*
Universität Würzburg, Institut für Anorganische Chemie, Am Hubland, D-97074 Würzburg, Germany
\end{abstract}

Supporting Information

* To whom correspondence should be addressed. Phone: +49-931-888-5250. Fax: +49-931-8884609. E-mail: r.tacke@mail.uni-wuerzburg.de. 


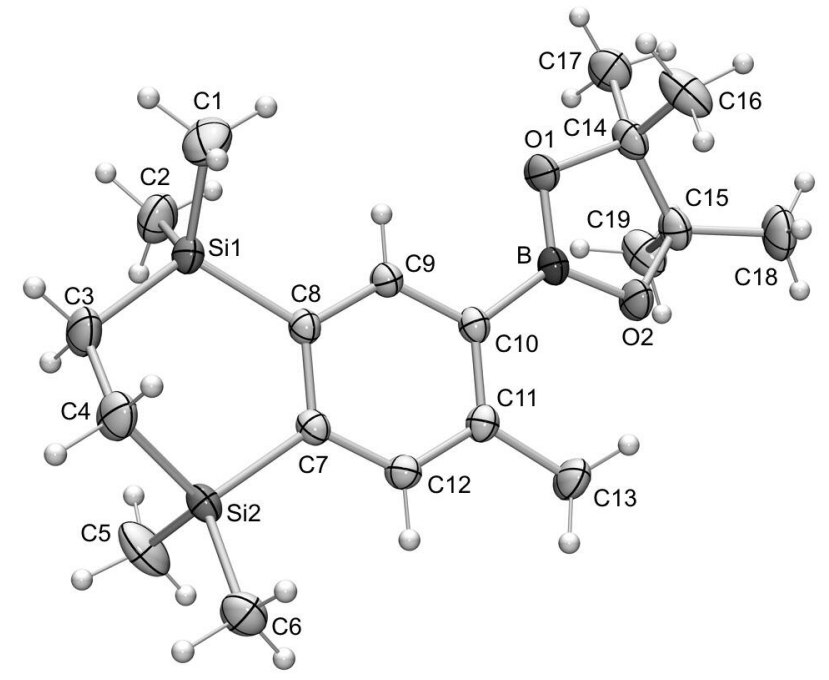

Figure S1. Molecular structure of $\mathbf{5}$ in the crystal (probability level of displacement ellipsoids $50 \%)$.
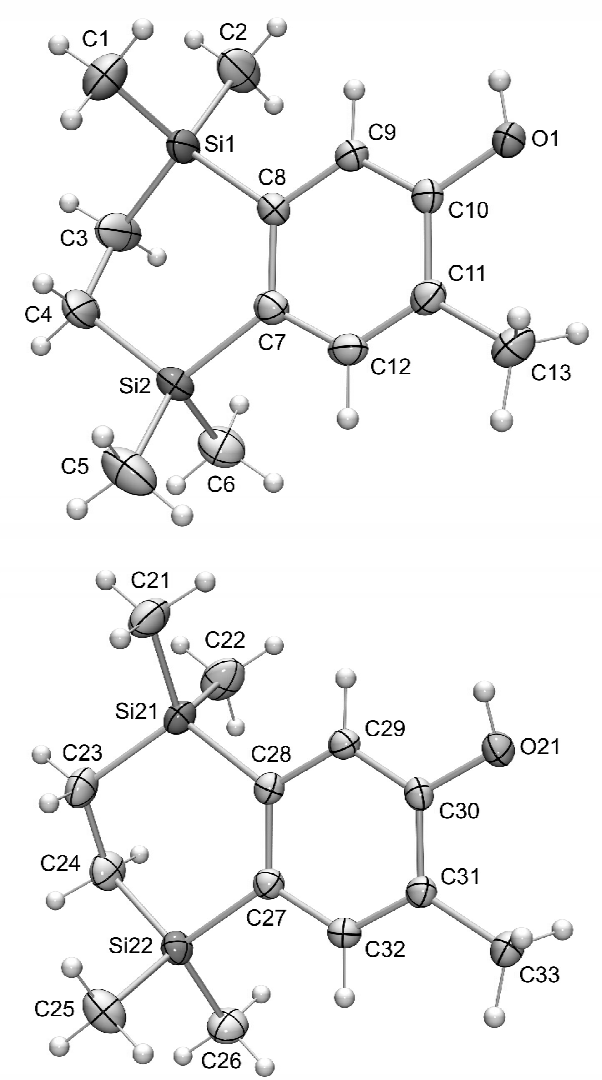

Figure S2. Molecular structures of the two crystallographically independent molecules in the crystal of 6 (probability level of displacement ellipsoids 50\%). 
Table s1. Crystal data and structure refinement for 5 .

\begin{tabular}{|c|c|}
\hline Identification code & 5 \\
\hline Empirical formula & $\mathrm{C}_{19} \mathrm{H}_{33} \mathrm{BO}_{2} \mathrm{Si}_{2}$ \\
\hline Formula weight & 360.44 \\
\hline Temperature & $193(2) \mathrm{K}$ \\
\hline Wavelength & $0.71073 \AA$ \\
\hline Crystal system, space group & Monoclinic, $\quad P 2_{1} / C$ \\
\hline Unit cell dimensions & $\begin{array}{l}a=15.5982(19) \AA \quad \alpha=90 \mathrm{deg} . \\
b=13.2100(15) \AA \quad \beta=108.847(12) \text { deg. } \\
c=11.1157(12) \AA \quad \gamma=90 \mathrm{deg} .\end{array}$ \\
\hline Volume & $2167.6(4) \AA^{3}$ \\
\hline Z, Calculated density & 4, $1.104 \mathrm{Mg} / \mathrm{m}^{3}$ \\
\hline Absorption coefficient & $0.172 \mathrm{~mm}^{-1}$ \\
\hline$F(000)$ & 784 \\
\hline Crystal size & $0.5 \times 0.5 \times 0.4 \mathrm{~mm}$ \\
\hline$\theta$ range for data collection & 3.08 to 27.95 deg. \\
\hline Limiting indices & $-20 \leq h \leq 20,-17 \leq k \leq 17,-14 \leq 1 \leq 14$ \\
\hline Reflections collected / unique & $24408 / 5141[R($ int $)=0.0695]$ \\
\hline Completeness to $\theta=27.95$ & $98.6 \%$ \\
\hline Absorption correction & None \\
\hline Refinement method & Full-matrix least-squares on $F^{2}$ \\
\hline Data / restraints / parameters & $5141 / 0 / 226$ \\
\hline Goodness-of-fit on $F^{2}$ & 1.038 \\
\hline Final $R$ indices $[I>2 \sigma(I)]$ & $R 1=0.0498, w R 2=0.1012$ \\
\hline$R$ indices (all data) & $R 1=0.0601, w R 2=0.1072$ \\
\hline
\end{tabular}




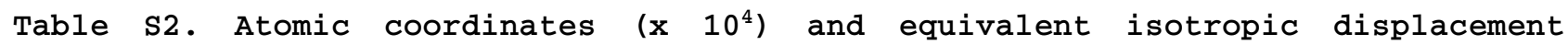
parameters $\left(\AA^{2} \times 10^{3}\right)$ for 5 . U(eq) is defined as one third of the trace of the orthogonalized Uij tensor.

\begin{tabular}{|c|c|c|c|c|}
\hline & $\mathrm{x}$ & y & $\mathrm{z}$ & $\mathrm{U}(\mathrm{eq})$ \\
\hline Si1 & $4292(1)$ & $5619(1)$ & $7074(1)$ & $30(1)$ \\
\hline Si2 & $2529(1)$ & $6872(1)$ & $7708(1)$ & $28(1)$ \\
\hline B & $1879(1)$ & $4160(1)$ & $2843(1)$ & $26(1)$ \\
\hline 01 & $2596(1)$ & $3656(1)$ & $2667(1)$ & $36(1)$ \\
\hline $\mathrm{O} 2$ & $1093(1)$ & $4047(1)$ & $1848(1)$ & $36(1)$ \\
\hline $\mathrm{C} 1$ & $4750(1)$ & $4319(2)$ & $7564(2)$ & $56(1)$ \\
\hline $\mathrm{C} 2$ & $4947(1)$ & $6231(2)$ & $6128(2)$ & $48(1)$ \\
\hline C3 & $4395(1)$ & $6401(2)$ & $8531(2)$ & $47(1)$ \\
\hline $\mathrm{C} 4$ & $3551(1)$ & $6366(2)$ & $8971(2)$ & $42(1)$ \\
\hline $\mathrm{C} 5$ & $1511(1)$ & $6890(1)$ & $8244(2)$ & $42(1)$ \\
\hline C6 & $2749(2)$ & $8186(1)$ & $7251(2)$ & $54(1)$ \\
\hline $\mathrm{C} 7$ & $2342(1)$ & $6020(1)$ & $6285(1)$ & $25(1)$ \\
\hline $\mathrm{C} 8$ & $3069(1)$ & $5512(1)$ & $6041(1)$ & $24(1)$ \\
\hline C9 & $2866(1)$ & $4912(1)$ & $4941(1)$ & $25(1)$ \\
\hline C10 & $1990(1)$ & $4806(1)$ & $4067(1)$ & $24(1)$ \\
\hline C11 & $1274(1)$ & $5318(1)$ & $4318(1)$ & $26(1)$ \\
\hline $\mathrm{C} 12$ & $1466(1)$ & $5900(1)$ & $5424(1)$ & $27(1)$ \\
\hline C13 & $310(1)$ & $5280(2)$ & $3429(2)$ & $41(1)$ \\
\hline C14 & $2234(1)$ & $3023(1)$ & $1535(1)$ & $34(1)$ \\
\hline C15 & $1337(1)$ & $3573(1)$ & $813(1)$ & $34(1)$ \\
\hline C16 & $2082(2)$ & $1963(1)$ & $1997(2)$ & $51(1)$ \\
\hline C17 & $2929(1)$ & $2978(2)$ & $843(2)$ & $47(1)$ \\
\hline C18 & $561(1)$ & $2892(2)$ & $70(2)$ & $53(1)$ \\
\hline C19 & $1455(2)$ & $4440(1)$ & $-28(2)$ & $47(1)$ \\
\hline
\end{tabular}


Table S3. Bond lengths [Å] and angles [deg] for 5 .

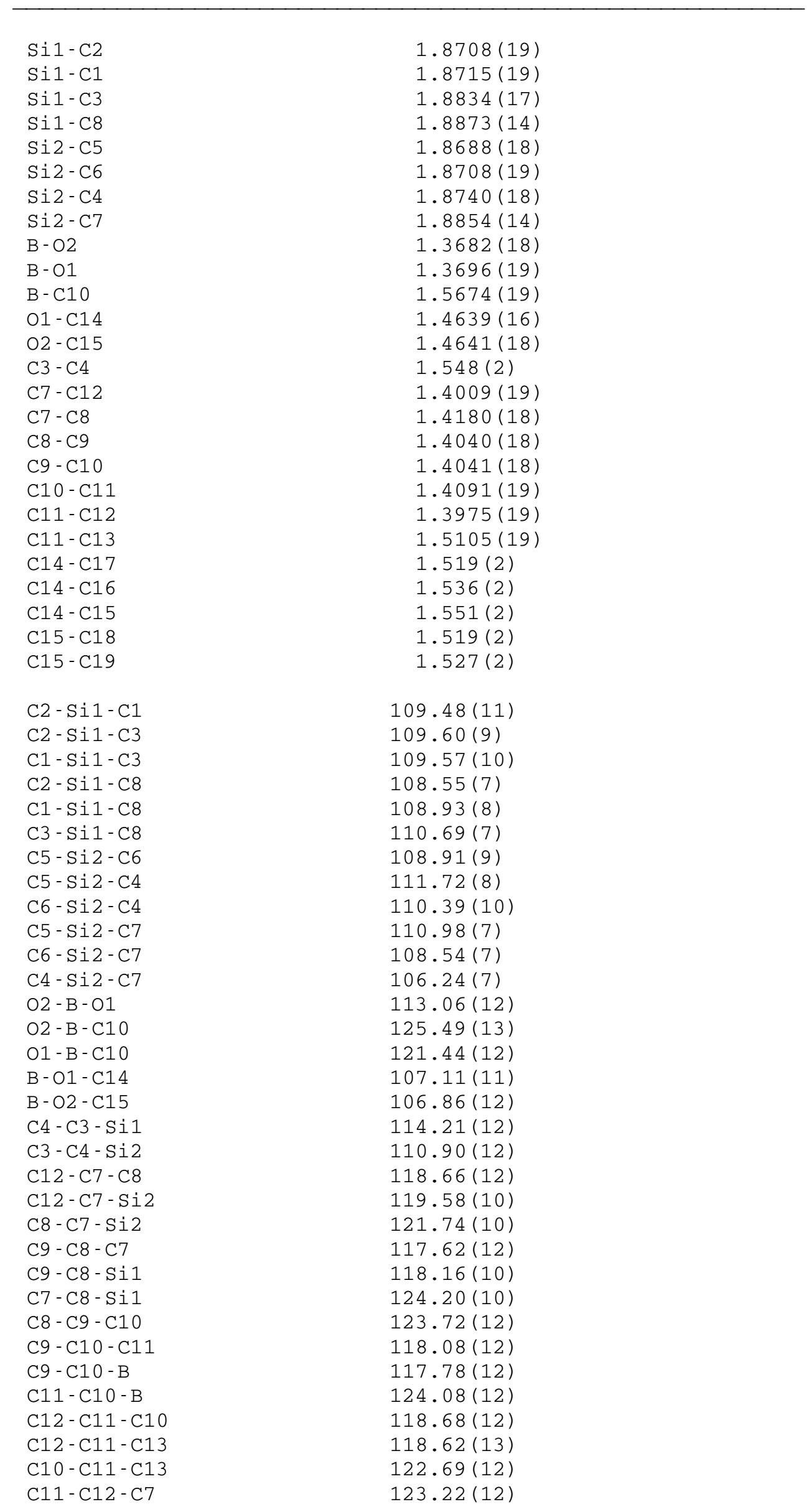


O1 - C14-C17

O1 - C14 - C16

C17-C14-C16

O1 - C14-C15

C1 1 - C14 - C15

C16 - C14 - C15

O2 - C15-C18

O2 - C15-C19

C18 - C15 - C19

O2 - C15 - C14

C18-C15-C14

C19-C15-C14
$108.50(13)$

$106.94(12)$

$110.26(15)$

$102.17(11)$

$115.40(14)$

$112.86(15)$

$108.50(14)$

$105.82(13)$

$110.17(15)$

$102.34(11)$

$115.53(15)$

$113.60(14)$ 
Table S4. Anisotropic displacement parameters $\left(\AA^{2} \times 10^{3}\right)$ for 5 . The anisotropic displacement factor exponent takes the form:

$-2 \pi^{2}\left[\mathrm{~h}^{2} \mathrm{a} *^{2} \mathrm{U} 11+\ldots+2 \mathrm{~h} k \mathrm{a}\right.$ * $\left.\mathrm{b} * \mathrm{U} 12\right]$

\begin{tabular}{|c|c|c|c|c|c|c|}
\hline & U11 & U2 2 & U33 & U23 & U13 & U12 \\
\hline Sil & $25(1)$ & $36(1)$ & $25(1)$ & $-6(1)$ & $2(1)$ & $1(1)$ \\
\hline Si2 & $35(1)$ & $27(1)$ & $23(1)$ & $-6(1)$ & $10(1)$ & $-1(1)$ \\
\hline B & $29(1)$ & $27(1)$ & $22(1)$ & $-2(1)$ & $6(1)$ & $-3(1)$ \\
\hline 01 & $34(1)$ & $43(1)$ & $25(1)$ & $-13(1)$ & $3(1)$ & $4(1)$ \\
\hline $\mathrm{O} 2$ & $32(1)$ & $47(1)$ & $25(1)$ & $-12(1)$ & $4(1)$ & $-2(1)$ \\
\hline $\mathrm{C} 1$ & $46(1)$ & $48(1)$ & $57(1)$ & $4(1)$ & $-8(1)$ & $12(1)$ \\
\hline $\mathrm{C} 2$ & $34(1)$ & $66(1)$ & $44(1)$ & $-8(1)$ & $13(1)$ & $-10(1)$ \\
\hline $\mathrm{C} 3$ & $33(1)$ & $70(1)$ & $34(1)$ & $-22(1)$ & $5(1)$ & $-5(1)$ \\
\hline $\mathrm{C} 4$ & $38(1)$ & $60(1)$ & $24(1)$ & $-9(1)$ & $5(1)$ & $1(1)$ \\
\hline $\mathrm{C} 5$ & $44(1)$ & $50(1)$ & $34(1)$ & $-8(1)$ & $17(1)$ & $2(1)$ \\
\hline $\mathrm{C} 6$ & $87(2)$ & $31(1)$ & $52(1)$ & $-10(1)$ & $33(1)$ & $-11(1)$ \\
\hline $\mathrm{C} 7$ & $29(1)$ & $23(1)$ & $21(1)$ & $0(1)$ & $6(1)$ & $1(1)$ \\
\hline $\mathrm{C} 8$ & $26(1)$ & $25(1)$ & $20(1)$ & $-1(1)$ & $5(1)$ & $0(1)$ \\
\hline C9 & $26(1)$ & $26(1)$ & $23(1)$ & $-3(1)$ & $6(1)$ & $1(1)$ \\
\hline C10 & $28(1)$ & $23(1)$ & $19(1)$ & $0(1)$ & $6(1)$ & $-2(1)$ \\
\hline C11 & $25(1)$ & $28(1)$ & $22(1)$ & $1(1)$ & $4(1)$ & $0(1)$ \\
\hline C12 & $27(1)$ & $29(1)$ & $24(1)$ & $-1(1)$ & $7(1)$ & $4(1)$ \\
\hline C13 & $26(1)$ & $57(1)$ & $33(1)$ & $-10(1)$ & $1(1)$ & $2(1)$ \\
\hline C14 & $44(1)$ & $33(1)$ & $23(1)$ & $-9(1)$ & $9(1)$ & $-1(1)$ \\
\hline C15 & $40(1)$ & $37(1)$ & $23(1)$ & $-9(1)$ & $7(1)$ & $-5(1)$ \\
\hline C16 & $84(1)$ & $31(1)$ & $38(1)$ & $-3(1)$ & $22(1)$ & $5(1)$ \\
\hline C17 & $49(1)$ & $55(1)$ & $39(1)$ & $-14(1)$ & $18(1)$ & $1(1)$ \\
\hline C18 & $51(1)$ & $62(1)$ & $38(1)$ & $-20(1)$ & $3(1)$ & $-16(1)$ \\
\hline C19 & $68(1)$ & $42(1)$ & $27(1)$ & $-1(1)$ & $10(1)$ & $3(1)$ \\
\hline
\end{tabular}


Table S5. Hydrogen coordinates $\left(x 10^{4}\right)$ and isotropic displacement parameters $\left(\AA^{2}\right.$ $x 10^{3}$ ) for 5 .

\begin{tabular}{|c|c|c|c|c|}
\hline & $\mathrm{x}$ & $y$ & z & $\mathrm{U}(\mathrm{eq})$ \\
\hline $\mathrm{H} 1 \mathrm{~A}$ & 4629 & 3889 & 6809 & 84 \\
\hline $\mathrm{H} 1 \mathrm{~B}$ & 4457 & 4030 & 8143 & 84 \\
\hline $\mathrm{H} 1 \mathrm{C}$ & 5405 & 4359 & 7996 & 84 \\
\hline $\mathrm{H} 2 \mathrm{~A}$ & 5597 & 6180 & 6593 & 72 \\
\hline H2B & 4775 & 6946 & 5989 & 72 \\
\hline $\mathrm{H} 2 \mathrm{C}$ & 4813 & 5888 & 5306 & 72 \\
\hline H3A & 4926 & 6161 & 9235 & 57 \\
\hline H3B & 4511 & 7114 & 8353 & 57 \\
\hline $\mathrm{H} 4 \mathrm{~A}$ & 3664 & 6773 & 9755 & 50 \\
\hline $\mathrm{H} 4 \mathrm{~B}$ & 3436 & 5658 & 9170 & 50 \\
\hline H5A & 1646 & 7279 & 9034 & 63 \\
\hline H5B & 1348 & 6196 & 8392 & 63 \\
\hline $\mathrm{H} 5 \mathrm{C}$ & 1003 & 7206 & 7587 & 63 \\
\hline H6A & 2193 & 8463 & 6648 & 81 \\
\hline H6B & 3226 & 8168 & 6853 & 81 \\
\hline $\mathrm{H} 6 \mathrm{C}$ & 2944 & 8613 & 8011 & 81 \\
\hline H9 & 3348 & 4559 & 4780 & 30 \\
\hline H12 & 979 & 6230 & 5600 & 32 \\
\hline H13A & -97 & 5557 & 3859 & 61 \\
\hline $\mathrm{H} 13 \mathrm{~B}$ & 142 & 4577 & 3183 & 61 \\
\hline $\mathrm{H} 13 \mathrm{C}$ & 258 & 5682 & 2668 & 61 \\
\hline H16A & 2645 & 1721 & 2619 & 76 \\
\hline H16B & 1897 & 1498 & 1272 & 76 \\
\hline $\mathrm{H} 16 \mathrm{C}$ & 1606 & 1995 & 2395 & 76 \\
\hline H17A & 3074 & 3666 & 644 & 70 \\
\hline H17B & 2681 & 2592 & 54 & 70 \\
\hline $\mathrm{H} 17 \mathrm{C}$ & 3480 & 2646 & 1386 & 70 \\
\hline $\mathrm{H} 18 \mathrm{~A}$ & 409 & 2424 & 656 & 79 \\
\hline $\mathrm{H} 18 \mathrm{~B}$ & 743 & 2505 & -559 & 79 \\
\hline $\mathrm{H} 18 \mathrm{C}$ & 31 & 3307 & -365 & 79 \\
\hline H19A & 904 & 4854 & -291 & 70 \\
\hline H19B & 1567 & 4164 & -782 & 70 \\
\hline H19C & 1971 & 4860 & 450 & 70 \\
\hline
\end{tabular}


Table s6. Torsion angles [deg] for 5 .

\begin{tabular}{|c|c|}
\hline O2 - B - O1 - C14 & $-9.01(17)$ \\
\hline C10 - B - O1 - C14 & $172.10(13)$ \\
\hline O1 - B - O2 - C15 & $-10.65(17)$ \\
\hline $\mathrm{C} 10-\mathrm{B}-\mathrm{O} 2-\mathrm{C} 15$ & $168.19(13)$ \\
\hline $\mathrm{C} 2-\mathrm{Si} 1-\mathrm{C} 3-\mathrm{C} 4$ & $148.04(15)$ \\
\hline C1-Si1-C3-C4 & $-91.81(17)$ \\
\hline C8-Si1-C3-C4 & $28.34(17)$ \\
\hline Si1-C3-C4-Si2 & $-61.19(18)$ \\
\hline $\mathrm{C} 5-\mathrm{Si} 2-\mathrm{C} 4-\mathrm{C} 3$ & $-177.47(12)$ \\
\hline $\mathrm{C} 6-\mathrm{Si} 2-\mathrm{C} 4-\mathrm{C} 3$ & $-56.13(15)$ \\
\hline $\mathrm{C} 7-\mathrm{Si} 2-\mathrm{C} 4-\mathrm{C} 3$ & $61.36(14)$ \\
\hline C5-Si2-C7-C12 & $30.56(13)$ \\
\hline $\mathrm{C} 6-\mathrm{Si} 2-\mathrm{C} 7-\mathrm{C} 12$ & $-89.08(14)$ \\
\hline C4-Si2-C7-C12 & $152.20(12)$ \\
\hline C5-Si2-C7-C8 & $-150.96(12)$ \\
\hline C6-Si2-C7-C8 & $89.39(14)$ \\
\hline C4-Si2-C7-C8 & $-29.32(13)$ \\
\hline $\mathrm{C} 12-\mathrm{C} 7-\mathrm{C} 8-\mathrm{C} 9$ & $-0.06(19)$ \\
\hline $\mathrm{Si} 2-\mathrm{C} 7-\mathrm{C} 8-\mathrm{C} 9$ & $-178.55(10)$ \\
\hline C12-C7-C8-Si1 & $178.25(10)$ \\
\hline Si2-C7-C8-Si1 & $-0.24(17)$ \\
\hline $\mathrm{C} 2-\mathrm{Si} 1-\mathrm{C} 8-\mathrm{C} 9$ & $62.59(13)$ \\
\hline C1-Si1-C8-C9 & $-56.55(14)$ \\
\hline C3-Si1-C8-C9 & $-177.08(12)$ \\
\hline $\mathrm{C} 2-\mathrm{Si} 1-\mathrm{C} 8-\mathrm{C} 7$ & $-115.71(13)$ \\
\hline C1-Si1-C8-C7 & $125.15(14)$ \\
\hline $\mathrm{C} 3-\mathrm{Si} 1-\mathrm{C} 8-\mathrm{C} 7$ & $4.62(15)$ \\
\hline C7 - C8 - C9 - C10 & $1.2(2)$ \\
\hline Si1-C8-C9-C10 & $-177.22(10)$ \\
\hline C8 - C9 - C10 - C11 & $-1.0(2)$ \\
\hline $\mathrm{C} 8-\mathrm{C} 9-\mathrm{C} 10-\mathrm{B}$ & $176.50(13)$ \\
\hline O2 - B - C10 - C9 & $-174.24(13)$ \\
\hline $\mathrm{O} 1-\mathrm{B}-\mathrm{C} 10-\mathrm{C} 9$ & $4.5(2)$ \\
\hline $\mathrm{O} 2-\mathrm{B}-\mathrm{C} 10-\mathrm{C} 11$ & $3.1(2)$ \\
\hline O1 - B - C10 - C11 & $-178.19(13)$ \\
\hline C9 - C10 - C11 - C12 & $-0.40(19)$ \\
\hline B - C10 - C11-C12 & $-177.70(13)$ \\
\hline C9 - C10 - C11 - C13 & $178.37(14)$ \\
\hline $\mathrm{B}-\mathrm{C} 10-\mathrm{C} 11-\mathrm{C} 13$ & $1.1(2)$ \\
\hline C10-C11-C12-C7 & $1.6(2)$ \\
\hline $\mathrm{C} 13-\mathrm{C} 11-\mathrm{C} 12-\mathrm{C} 7$ & $-177.26(14)$ \\
\hline C8 - C7 - C12-C11 & $-1.3(2)$ \\
\hline Si2-C7-C12-C11 & $177.21(11)$ \\
\hline B - O1 - C14 - C17 & $145.58(14)$ \\
\hline $\mathrm{B}-\mathrm{O} 1-\mathrm{C} 14-\mathrm{C} 16$ & $-95.50(16)$ \\
\hline B - O1-C14-C15 & $23.26(15)$ \\
\hline B - O2 - C15-C18 & $146.80(15)$ \\
\hline B - O2 - C15-C19 & $-94.99(15)$ \\
\hline B - O2 - C15-C14 & $24.22(15)$ \\
\hline O1 - C14 - C15 - O2 & $-28.49(14)$ \\
\hline $\mathrm{C} 17-\mathrm{C} 14-\mathrm{C} 15-\mathrm{O} 2$ & $-145.98(13)$ \\
\hline C16-C14-C15-O2 & $85.99(14)$ \\
\hline $\mathrm{O} 1-\mathrm{C} 14-\mathrm{C} 15-\mathrm{C} 18$ & $-146.17(14)$ \\
\hline C17-C14-C15-C18 & $96.33(18)$ \\
\hline C16-C14-C15- C18 & $-31.70(19)$ \\
\hline O1 - C14 - C15 - C19 & $85.10(15)$ \\
\hline C17 - C14 - C15 - C19 & $-32.39(19)$ \\
\hline C16-C14 - C15 - C19 & $-160.42(13)$ \\
\hline
\end{tabular}


Table s7. Crystal data and structure refinement for 6 .

\begin{tabular}{|c|c|}
\hline Identification code & 6 \\
\hline Empirical formula & $\mathrm{C}_{13} \mathrm{H}_{22} \mathrm{OSi}_{2}$ \\
\hline Formula weight & 250.49 \\
\hline Temperature & $173(2) \mathrm{K}$ \\
\hline Wavelength & $0.71073 \AA$ \\
\hline Crystal system, space group & Triclinic, $\quad P \overline{1}$ \\
\hline Unit cell dimensions & $\begin{array}{lll}a=9.2211(18) \AA & \alpha=92.22(3) \mathrm{deg} . \\
b=10.489(2) \AA & \beta=102.27(3) \mathrm{deg} . \\
c=16.445(3) \AA & \gamma=100.55(3) \mathrm{deg} .\end{array}$ \\
\hline Volume & $1522.9(5) \AA^{3}$ \\
\hline Z, Calculated density & $4, \quad 1.093 \mathrm{Mg} / \mathrm{m}^{3}$ \\
\hline Absorption coefficient & $0.214 \mathrm{~mm}^{-1}$ \\
\hline$F(000)$ & 544 \\
\hline Crystal size & $0.3 \times 0.3 \times 0.1 \mathrm{~mm}$ \\
\hline$\theta$ range for data collection & 2.27 to $28.00 \mathrm{deg}$ \\
\hline Limiting indices & $-12 \leq h \leq 12,-13 \leq k \leq 13,-21 \leq 1 \leq 21$ \\
\hline Reflections collected / unique & $22192 / 6771[R($ int $)=0.0400]$ \\
\hline Completeness to $\theta=28.00$ & $92.1 \%$ \\
\hline Absorption correction & None \\
\hline Refinement method & Full-matrix least-squares on $F^{2}$ \\
\hline Data / restraints / parameters & $6771 / 0 / 301$ \\
\hline Goodness-of-fit on $F^{2}$ & 1.027 \\
\hline Final $R$ indices $[I>2 \sigma(I)]$ & $R 1=0.0413, w R 2=0.1022$ \\
\hline$R$ indices (all data) & $R 1=0.0654, w R 2=0.1132$ \\
\hline Largest diff. peak and hole & 0.546 and -0.587 e $\AA^{-3}$ \\
\hline
\end{tabular}




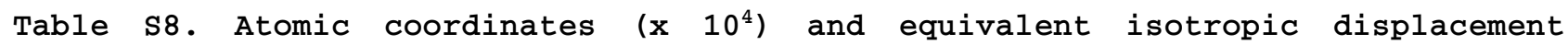
parameters $\left(\AA^{2} \times 10^{3}\right)$ for 6 . U(eq) is defined as one third of the trace of the orthogonalized Uij tensor.

\begin{tabular}{|c|c|c|c|c|}
\hline & $\mathrm{x}$ & $\mathrm{y}$ & $\mathrm{z}$ & $\mathrm{U}(\mathrm{eq})$ \\
\hline Sil & $6409(1)$ & $4093(1)$ & $1361(1)$ & $38(1)$ \\
\hline Si2 & $8331(1)$ & $1924(1)$ & $557(1)$ & $33(1)$ \\
\hline $\mathrm{C} 1$ & $4499(3)$ & $3250(3)$ & $1466(2)$ & $65(1)$ \\
\hline $\mathrm{C} 2$ & $6710(3)$ & $5848(3)$ & $1729(2)$ & $55(1)$ \\
\hline $\mathrm{C} 3$ & $6610(3)$ & $3910(3)$ & $253(1)$ & $58(1)$ \\
\hline $\mathrm{C} 4$ & $6613(3)$ & $2478(3)$ & $-14(1)$ & $57(1)$ \\
\hline $\mathrm{C} 5$ & $8088(3)$ & $114(2)$ & $392(2)$ & $57(1)$ \\
\hline $\mathrm{C} 6$ & $10012(3)$ & $2717(2)$ & $168(1)$ & $47(1)$ \\
\hline $\mathrm{C7}$ & $8641(2)$ & $2415(2)$ & $1705(1)$ & $27(1)$ \\
\hline $\mathrm{C} 8$ & $7864(2)$ & $3302(2)$ & $2022(1)$ & $26(1)$ \\
\hline C9 & $8178(2)$ & $3596(2)$ & $2892(1)$ & $25(1)$ \\
\hline C10 & $9190(2)$ & $3032(2)$ & $3434(1)$ & $23(1)$ \\
\hline $\mathrm{C} 11$ & $9965(2)$ & $2151(2)$ & $3139(1)$ & $26(1)$ \\
\hline $\mathrm{C} 12$ & $9675(2)$ & $1887(2)$ & $2276(1)$ & $29(1)$ \\
\hline C13 & $10997(2)$ & $1488(2)$ & $3731(1)$ & $39(1)$ \\
\hline 01 & $9464(1)$ & $3301(1)$ & $4290(1)$ & $27(1)$ \\
\hline $\operatorname{Si} 21$ & $5780(1)$ & $9102(1)$ & $3418(1)$ & $27(1)$ \\
\hline $\operatorname{Si} 22$ & $2329(1)$ & $6882(1)$ & $2824(1)$ & $27(1)$ \\
\hline 021 & $7950(1)$ & $5179(1)$ & $4735(1)$ & $28(1)$ \\
\hline $\mathrm{C} 21$ & $6984(3)$ & $10129(2)$ & $4364(1)$ & $42(1)$ \\
\hline $\mathrm{C} 22$ & $6703(3)$ & $9326(2)$ & $2513(2)$ & $47(1)$ \\
\hline $\mathrm{C} 23$ & $3865(2)$ & $9534(2)$ & $3141(1)$ & $35(1)$ \\
\hline $\mathrm{C} 24$ & $2751(2)$ & $8558(2)$ & $2459(1)$ & $36(1)$ \\
\hline $\mathrm{C} 25$ & $1025(2)$ & $6873(2)$ & $3558(1)$ & $41(1)$ \\
\hline $\mathrm{C} 26$ & $1424(2)$ & $5660(2)$ & $1917(1)$ & $39(1)$ \\
\hline $\mathrm{C} 27$ & $4148(2)$ & $6440(2)$ & $3392(1)$ & $23(1)$ \\
\hline $\mathrm{C} 28$ & $5536(2)$ & $7339(2)$ & $3656(1)$ & $23(1)$ \\
\hline $\mathrm{C} 29$ & $6803(2)$ & $6899(2)$ & $4095(1)$ & $23(1)$ \\
\hline $\mathrm{C} 30$ & $6717(2)$ & $5616(2)$ & $4284(1)$ & $22(1)$ \\
\hline C31 & $5374(2)$ & $4694(2)$ & $4017(1)$ & $22(1)$ \\
\hline C32 & $4124(2)$ & $5139(2)$ & $3578(1)$ & $24(1)$ \\
\hline C33 & $5311(2)$ & $3296(2)$ & $4203(1)$ & $30(1)$ \\
\hline
\end{tabular}


Table S9. Bond lengths [Å] and angles [deg] for 6 .

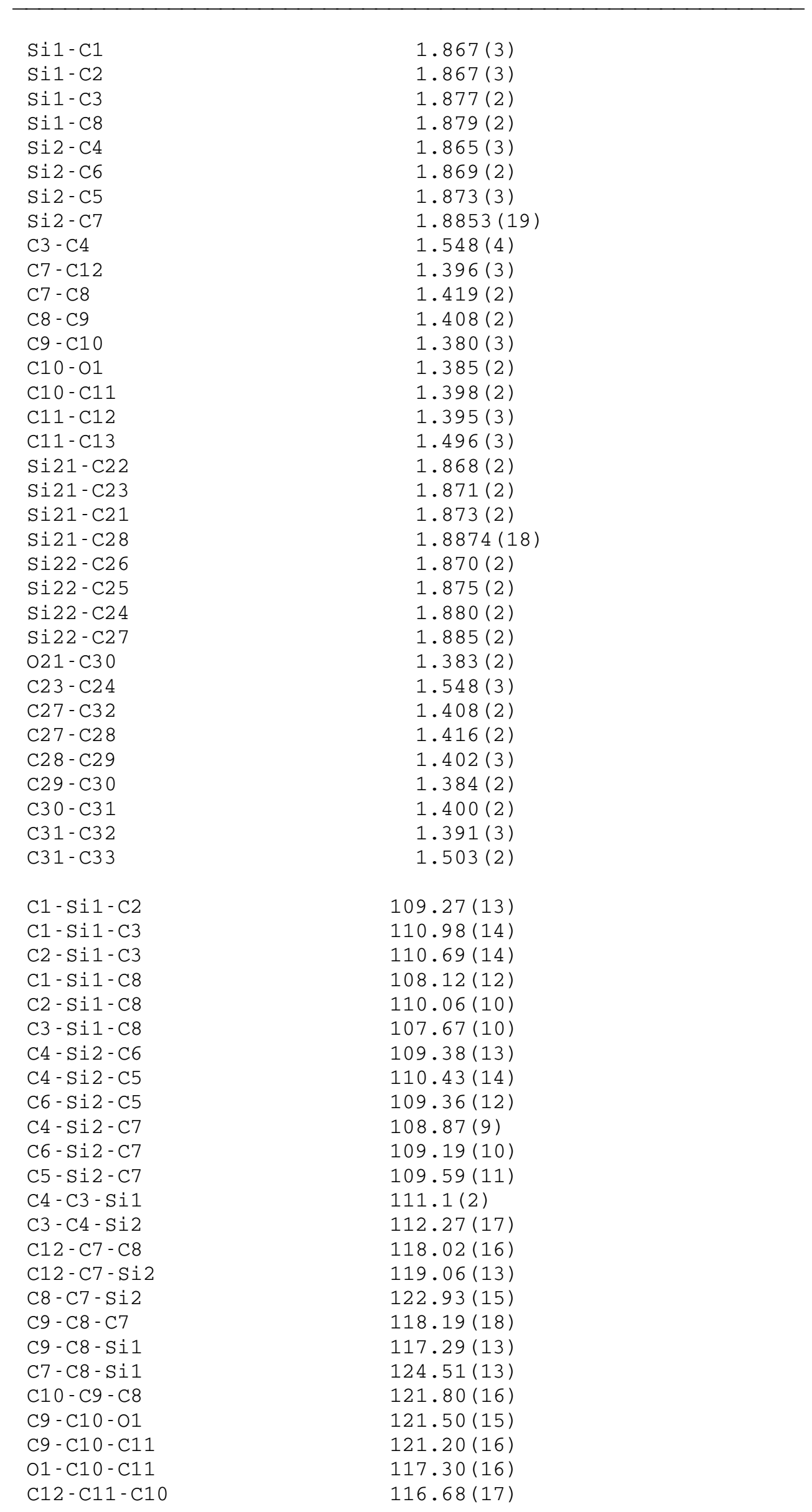




\begin{tabular}{ll} 
C12-C11-C13 & $122.30(17)$ \\
C10-C11-C13 & $120.96(17)$ \\
C11-C12-C7 & $124.08(17)$ \\
C22-Si21-C23 & $109.36(11)$ \\
C22-Si21-C21 & $110.31(11)$ \\
C23-Si21-C21 & $110.80(10)$ \\
C22-Si21-C28 & $109.24(9)$ \\
C23-Si21-C28 & $108.34(9)$ \\
C21-Si21-C28 & $108.75(9)$ \\
C26-Si22-C25 & $109.60(10)$ \\
C26-Si22-C24 & $110.52(10)$ \\
C25-Si22-C24 & $109.16(11)$ \\
C26-Si22-C27 & $109.19(9)$ \\
C25-Si22-C27 & $109.11(9)$ \\
C24-Si22-C27 & $109.23(9)$ \\
C24-C23-Si21 & $112.28(14)$ \\
C23-C24-Si22 & $112.33(14)$ \\
C32-C27-C28 & $117.76(16)$ \\
C32-C27-Si22 & $118.07(13)$ \\
C28-C27-Si22 & $124.16(13)$ \\
C29-C28-C27 & $118.81(16)$ \\
C29-C28-Si21 & $118.09(13)$ \\
C27-C28-Si21 & $123.08(14)$ \\
C30-C29-C28 & $121.45(15)$ \\
O21-C30-C29 & $121.91(15)$ \\
O21-C30-C31 & $116.77(15)$ \\
C29-C30-C31 & $121.31(16)$ \\
C32-C31-C30 & $116.81(16)$ \\
C32-C31-C33 & $122.50(15)$ \\
C30-C31-C33 & $120.69(16)$ \\
C31-C32-C27 & $123.82(16)$ \\
& \\
\hline & \\
C2 23$)$
\end{tabular}


Table S10. Anisotropic displacement parameters $\left(\AA^{2} \times 10^{3}\right)$ for 6 . The anisotropic displacement factor exponent takes the form:

$-2 \pi^{2}\left[\mathrm{~h}^{2} \mathrm{a} *^{2} \mathrm{U} 11+\ldots+2 \mathrm{~h} \mathrm{k} a * \mathrm{~b} * \mathrm{U} 12\right]$

\begin{tabular}{|c|c|c|c|c|c|c|}
\hline & U11 & U2 2 & U3 3 & U2 3 & U13 & U12 \\
\hline Sil & $37(1)$ & $51(1)$ & $25(1)$ & $-6(1)$ & $-3(1)$ & $24(1)$ \\
\hline Si2 & $36(1)$ & $36(1)$ & $28(1)$ & $-8(1)$ & $7(1)$ & $9(1)$ \\
\hline C1 & $34(1)$ & $88(2)$ & $68(2)$ & $-16(2)$ & $-1(1)$ & $22(1)$ \\
\hline $\mathrm{C} 2$ & $74(2)$ & $55(2)$ & $39(1)$ & $-1(1)$ & $-6(1)$ & $40(1)$ \\
\hline $\mathrm{C} 3$ & $70(2)$ & $85(2)$ & $26(1)$ & $0(1)$ & $-1(1)$ & $52(2)$ \\
\hline $\mathrm{C} 4$ & $51(1)$ & $87(2)$ & $31(1)$ & $-22(1)$ & $-4(1)$ & $29(1)$ \\
\hline $\mathrm{C} 5$ & $74(2)$ & $43(1)$ & $52(1)$ & $-14(1)$ & $21(1)$ & $3(1)$ \\
\hline $\mathrm{C} 6$ & $54(1)$ & $50(1)$ & $40(1)$ & $3(1)$ & $19(1)$ & $11(1)$ \\
\hline $\mathrm{C} 7$ & $26(1)$ & $26(1)$ & $29(1)$ & $-2(1)$ & $7(1)$ & $3(1)$ \\
\hline $\mathrm{C} 8$ & $25(1)$ & 29 (1) & $24(1)$ & $-4(1)$ & $4(1)$ & $6(1)$ \\
\hline $\mathrm{C} 9$ & $23(1)$ & $27(1)$ & $27(1)$ & $-1(1)$ & $4(1)$ & $8(1)$ \\
\hline C10 & $21(1)$ & $24(1)$ & $24(1)$ & $-1(1)$ & $3(1)$ & $3(1)$ \\
\hline C11 & $21(1)$ & 25 (1) & $33(1)$ & $1(1)$ & $4(1)$ & $5(1)$ \\
\hline C12 & $27(1)$ & $26(1)$ & $36(1)$ & $-3(1)$ & $9(1)$ & $8(1)$ \\
\hline C13 & $34(1)$ & $46(1)$ & $41(1)$ & $5(1)$ & $3(1)$ & $21(1)$ \\
\hline 01 & $26(1)$ & 31 (1) & $24(1)$ & $-1(1)$ & $0(1)$ & $11(1)$ \\
\hline Si21 & $33(1)$ & $20(1)$ & $30(1)$ & $3(1)$ & $7(1)$ & $5(1)$ \\
\hline Si22 & $25(1)$ & $29(1)$ & $26(1)$ & $3(1)$ & $3(1)$ & $8(1)$ \\
\hline O21 & $23(1)$ & $26(1)$ & $32(1)$ & $-1(1)$ & $0(1)$ & $8(1)$ \\
\hline $\mathrm{C} 21$ & $48(1)$ & $25(1)$ & $48(1)$ & $0(1)$ & $-1(1)$ & $4(1)$ \\
\hline $\mathrm{C} 22$ & $58(1)$ & $35(1)$ & $52(1)$ & $11(1)$ & $26(1)$ & $1(1)$ \\
\hline C23 & $44(1)$ & $26(1)$ & $37(1)$ & $4(1)$ & $5(1)$ & $13(1)$ \\
\hline $\mathrm{C} 24$ & $38(1)$ & $34(1)$ & $33(1)$ & $8(1)$ & $1(1)$ & $12(1)$ \\
\hline C25 & $32(1)$ & $54(1)$ & $39(1)$ & $6(1)$ & $9(1)$ & $13(1)$ \\
\hline C26 & $36(1)$ & 39 (1) & $35(1)$ & $-1(1)$ & $-2(1)$ & $4(1)$ \\
\hline $\mathrm{C} 27$ & $24(1)$ & $22(1)$ & $24(1)$ & $1(1)$ & $6(1)$ & $4(1)$ \\
\hline $\mathrm{C} 28$ & $25(1)$ & $22(1)$ & $22(1)$ & $2(1)$ & $6(1)$ & $5(1)$ \\
\hline C29 & $22(1)$ & $22(1)$ & $26(1)$ & $-1(1)$ & $6(1)$ & $3(1)$ \\
\hline C3O & $21(1)$ & $23(1)$ & $22(1)$ & $1(1)$ & $5(1)$ & $8(1)$ \\
\hline C31 & $23(1)$ & $20(1)$ & $25(1)$ & $1(1)$ & $8(1)$ & $5(1)$ \\
\hline C32 & $22(1)$ & $23(1)$ & $27(1)$ & $1(1)$ & $5(1)$ & $3(1)$ \\
\hline C33 & $30(1)$ & $22(1)$ & $39(1)$ & $4(1)$ & $9(1)$ & $6(1)$ \\
\hline
\end{tabular}




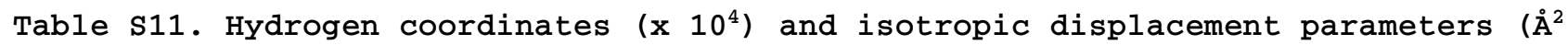
$x 10^{3}$ ) for 6 .

\begin{tabular}{|c|c|c|c|c|}
\hline & $\mathrm{x}$ & $\mathrm{y}$ & z & $\mathrm{U}(\mathrm{eq})$ \\
\hline $\mathrm{H} 1 \mathrm{~A}$ & 3721 & 3649 & 1126 & 97 \\
\hline H1B & 4434 & 3330 & 2053 & 97 \\
\hline $\mathrm{H} 1 \mathrm{C}$ & 4338 & 2327 & 1276 & 97 \\
\hline $\mathrm{H} 2 \mathrm{~A}$ & 7702 & 6298 & 1661 & 83 \\
\hline H2B & 6670 & 5925 & 2319 & 83 \\
\hline $\mathrm{H} 2 \mathrm{C}$ & 5917 & 6242 & 1398 & 83 \\
\hline H3A & 5762 & 4201 & -121 & 69 \\
\hline H3B & 7566 & 4470 & 196 & 69 \\
\hline $\mathrm{H} 4 \mathrm{~A}$ & 6564 & 2388 & -621 & 68 \\
\hline H4B & 5701 & 1912 & 94 & 68 \\
\hline H5A & 7233 & -304 & 617 & 85 \\
\hline H5B & 9012 & -159 & 679 & 85 \\
\hline H5C & 7890 & -142 & -207 & 85 \\
\hline H6A & 9880 & 2427 & -422 & 70 \\
\hline H6B & 10927 & 2476 & 495 & 70 \\
\hline $\mathrm{H} 6 \mathrm{C}$ & 10109 & 3664 & 226 & 70 \\
\hline H9 & 7679 & 4199 & 3112 & 31 \\
\hline H12 & 10213 & 1311 & 2062 & 35 \\
\hline H13A & 11802 & 2142 & 4084 & 59 \\
\hline $\mathrm{H} 13 \mathrm{~B}$ & 11444 & 916 & 3413 & 59 \\
\hline $\mathrm{H} 13 \mathrm{C}$ & 10419 & 970 & 4082 & 59 \\
\hline H1 & 8920 & 3816 & 4399 & 41 \\
\hline H2 & 8608 & 5818 & 4979 & 41 \\
\hline $\mathrm{H} 21 \mathrm{~A}$ & 7953 & 9844 & 4523 & 64 \\
\hline H21B & 6463 & 10037 & 4825 & 64 \\
\hline $\mathrm{H} 21 \mathrm{C}$ & 7166 & 11042 & 4237 & 64 \\
\hline H22A & 6846 & 10243 & 2393 & 71 \\
\hline H2 2B & 6059 & 8788 & 2022 & 71 \\
\hline $\mathrm{H} 22 \mathrm{C}$ & 7688 & 9064 & 2648 & 71 \\
\hline H23A & 3447 & 9551 & 3647 & 42 \\
\hline H23B & 3964 & 10416 & 2940 & 42 \\
\hline $\mathrm{H} 24 \mathrm{~A}$ & 3190 & 8512 & 1961 & 43 \\
\hline $\mathrm{H} 24 \mathrm{~B}$ & 1794 & 8879 & 2291 & 43 \\
\hline $\mathrm{H} 25 \mathrm{~A}$ & 57 & 7059 & 3258 & 61 \\
\hline H25B & 1482 & 7540 & 4020 & 61 \\
\hline H25C & 852 & 6017 & 3777 & 61 \\
\hline H26A & 1213 & 4793 & 2118 & 59 \\
\hline H26B & 2110 & 5663 & 1536 & 59 \\
\hline H26C & 476 & 5884 & 1621 & 59 \\
\hline H29 & 7741 & 7494 & 4267 & 28 \\
\hline H32 & 3201 & 4528 & 3393 & 29 \\
\hline H33A & 4276 & 2804 & 3999 & 45 \\
\hline H33B & 5620 & 3246 & 4807 & 45 \\
\hline H33 C & 5998 & 2925 & 3925 & 45 \\
\hline
\end{tabular}


Table S12. Torsion angles [deg] for 6 .

\begin{tabular}{|c|c|}
\hline $\mathrm{C} 1-\mathrm{Si} 1-\mathrm{C} 3-\mathrm{C} 4$ & $-67.4(2)$ \\
\hline $\mathrm{C} 2-\mathrm{Si} 1-\mathrm{C} 3-\mathrm{C} 4$ & $171.09(18)$ \\
\hline C8-Si1-C3-C4 & $50.7(2)$ \\
\hline Si1-C3-C4-Si2 & $-67.3(2)$ \\
\hline C6-Si2-C4-C3 & $-72.0(2)$ \\
\hline $\mathrm{C} 5-\mathrm{Si} 2-\mathrm{C} 4-\mathrm{C} 3$ & $167.61(18)$ \\
\hline C7 - Si2-C4-C3 & $47.2(2)$ \\
\hline C4-Si2-C7-C12 & $167.40(16)$ \\
\hline C6-Si2-C7 - C12 & $-73.24(17)$ \\
\hline C5-Si2-C7-C12 & $46.52(18)$ \\
\hline C4-Si2-C7-C8 & $-12.38(19)$ \\
\hline C6-Si2-C7 - C8 & $106.97(17)$ \\
\hline C5-Si2-C7-C8 & $-133.26(17)$ \\
\hline C12 - C7 - C8 - C9 & $-0.1(3)$ \\
\hline Si2-C7 - C8 - C9 & $179.73(13)$ \\
\hline C12-C7-C8-Si1 & $-178.86(13)$ \\
\hline Si2-C7-C8-Si1 & $0.9(2)$ \\
\hline C1-Si1-C8 - C9 & $-75.45(17)$ \\
\hline $\mathrm{C} 2-\mathrm{Si} 1-\mathrm{C} 8-\mathrm{C} 9$ & $43.84(18)$ \\
\hline C3-Si1-C8-C9 & $164.59(16)$ \\
\hline C1-Si1-C8-C7 & $103.37(18)$ \\
\hline $\mathrm{C} 2-\mathrm{Si} 1-\mathrm{C} 8-\mathrm{C} 7$ & $-137.35(17)$ \\
\hline C3-Si1-C8-C7 & $-16.6(2)$ \\
\hline C7 - C8 - C9 - C10 & $-1.1(3)$ \\
\hline Si1-C8-C9-C10 & $177.79(13)$ \\
\hline C8-C9-C10-O1 & $-178.31(16)$ \\
\hline C8 - C9 - C10-C11 & $0.8(3)$ \\
\hline C9 - C10 - C11 - C12 & $0.7(2)$ \\
\hline O1 - C10 - C11 - C12 & $179.81(15)$ \\
\hline C9 - C10 - C11 - C13 & $-176.64(17)$ \\
\hline O1 - C10 - C11 - C13 & $2.5(2)$ \\
\hline C10 - C11-C12 - C7 & $-1.9(3)$ \\
\hline C13-C11-C12-C7 & $175.37(18)$ \\
\hline C8 - C7 - C12 - C11 & $1.6(3)$ \\
\hline Si2-C7 - C12-C11 & $-178.20(14)$ \\
\hline C22-Si21-C23-C24 & $67.82(17)$ \\
\hline C21-Si21-C23-C24 & $-170.37(14)$ \\
\hline C28-Si21-C23-C24 & $-51.15(16)$ \\
\hline Si21-C23-C24-Si22 & $64.85(17)$ \\
\hline $\mathrm{C} 26-\mathrm{Si} 22-\mathrm{C} 24-\mathrm{C} 23$ & $-164.80(15)$ \\
\hline C25-Si22-C24-C23 & $74.59(17)$ \\
\hline C27-Si22-C24-C23 & $-44.65(17)$ \\
\hline C26-Si22-C27-C32 & $-47.28(16)$ \\
\hline C25-Si22-C27-C32 & $72.49(16)$ \\
\hline C24-Si22-C27-C32 & $-168.25(13)$ \\
\hline C26-Si22-C27-C28 & $133.83(15)$ \\
\hline C25-Si22-C27-C28 & $-106.41(16)$ \\
\hline C24-Si22-C27-C28 & $12.85(17)$ \\
\hline C32 - C2 7- C28-C29 & $-0.8(2)$ \\
\hline Si22-C27-C28-C29 & $178.13(12)$ \\
\hline C32-C27-C28-Si21 & $177.43(12)$ \\
\hline Si22-C27-C28-Si21 & $-3.7(2)$ \\
\hline C22-Si21-C28-C29 & $78.25(16)$ \\
\hline C23-Si21-C28-C29 & $-162.71(13)$ \\
\hline C21-Si21-C28-C29 & $-42.20(16)$ \\
\hline C22-Si21-C28-C27 & $-99.97(16)$ \\
\hline C23-Si21-C28-C27 & $19.08(16)$ \\
\hline C21-Si21-C28-C27 & $139.58(15)$ \\
\hline C2 $7-C 28-C 29-C 30$ & $-1.0(2)$ \\
\hline $\mathrm{Si} 21-\mathrm{C} 28-\mathrm{C} 29-\mathrm{C} 30$ & $-179.26(12)$ \\
\hline C28 - C29-C30-O21 & $-178.31(14)$ \\
\hline
\end{tabular}


C2 $8-C 29-\mathrm{C} 30-\mathrm{C} 31$

$\mathrm{O} 21-\mathrm{C} 30-\mathrm{C} 31-\mathrm{C} 32$

C29-C30-C31-C32

$\mathrm{O} 21-\mathrm{C} 30-\mathrm{C} 31-\mathrm{C} 33$

C29-C30-C31-C33

C30 - C31 - C32 - C2 7

C33 - C31-C32-C2 7

C2 8-C2 $7-$ C32 - C31

Si22-C2 7 - C32-C31
$2.4(2)$

$178.70(14)$

$-2.0(2)$

$-1.3(2)$

$178.00(15)$

$0.2(2)$

$-179.80(15)$

$1.2(2)$

$-177.81(13)$ 